\title{
Open-Source Miniature Fluorimeter to Monitor Real- Time Isothermal Nucleic Acid Amplification Reactions in Resource-Limited Settings
}

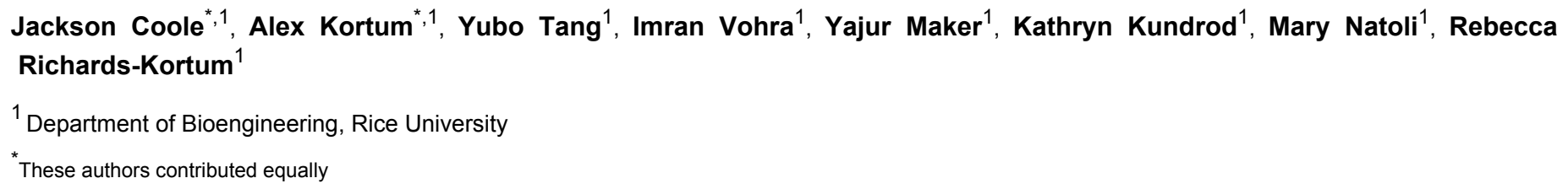

\section{Corresponding Author}

Rebecca Richards-Kortum rkortum@rice.edu

\section{Citation}

Coole, J., Kortum, A., Tang, Y., Vohra, I., Maker, Y., Kundrod, K., Natoli, M., Richards-Kortum, R. Open-Source Miniature Fluorimeter to Monitor Real-Time Isothermal Nucleic Acid Amplification Reactions in ResourceLimited Settings. J. Vis. Exp. (168), e62148, doi:10.3791/62148 (2021).

\section{Date Published}

February 3, 2021

DOI

$10.3791 / 62148$

URL

jove.com/video/62148

\section{Abstract}

Traditional methods to detect and quantify nucleic acids rely on polymerase chain reaction $(P C R)$ and require the use of expensive thermocyclers with integrated fluorescence detection of amplicons. Isothermal nucleic acid amplification technologies eliminate the need for thermal cycling; however, fluorescence-based detection of products is still required for real-time, quantitative results. Several portable isothermal heaters with integrated fluorescence detection are now commercially available; however, the cost of these devices remains a significant barrier to widespread adoption in resource-limited settings. Described here is a protocol for the design and assembly of a modular, low-cost fluorimeter constructed from off-the-shelf components. Enclosed in a compact 3D printed housing, the fluorimeter is designed to be placed atop a commercially available heat block holding a PCR tube. The fluorimeter described here was optimized to detect fluorescein isothiocyanate (FITC) dye, but the system can be modified for use with dyes commonly used as reporters in real-time nucleic acid amplification reactions. Clinical applicability of the system is demonstrated by performing real-time nucleic acid detection with two isothermal amplification technologies: recombinase polymerase amplification (RPA) for detection of positive control DNA provided in a commercial kit and reverse transcription loopmediated isothermal amplification (RT-LAMP) for detection of clinically meaningful levels of SARS-CoV-2 RNA.

\section{Introduction}

Isothermal amplification technologies are widely used for detection of nucleic acids. Compared to traditional
PCR approaches that require thermocycling, isothermal amplification allows nucleic acid amplification to occur at 
a single temperature, thus enabling faster time-to-results and better tolerance of inhibitors ${ }^{1,2}$. Another key benefit of isothermal amplification is the reduced instrumentation complexity. Most isothermal amplification reactions only require a heat block and a detection modality-either real-time detection via fluorescence monitoring or endpoint detection, for example by lateral flow or gel electrophoresis ${ }^{3,4}$. Realtime fluorescence detection is accomplished through the detection of fluorescence produced by intercalating dyes that activate in the presence of double-stranded DNA or quenched fluorescent probes that activate in the presence of specific double-stranded DNA sequences.

While commercially available benchtop isothermal fluorimeters exist, many lack customization for assay implementation. For example, many devices require specific or company-provided consumables, recommend preferred vendors, or utilize proprietary software to obtain advertised results. Most of these systems cost over \$5,000 USD, representing a significant barrier for widespread use in resource-limited settings. In addition, users in low-resource settings face challenges to maintain equipment designed for high-resource settings due to harsh environmental conditions, weak supply chains for spare parts, and specialized tools required for maintenance and repair $^{5}$. To meet this need, described here is the design and assembly of a modular and low-cost fluorimeter constructed from off-theshelf components enclosed in a compact 3D printed housing (Figures 1A-C) with two optional configurations. The first configuration of this device uses commercially available glass filters and a dichroic mirror to block excess background light and has a total cost of assembly of $\$ 830$ USD. While these filters are commonly used in fluorescence-based imaging systems, replacing expensive high-grade optical filter foils has previously been shown to allow for nucleic acid detection ${ }^{6}$. The second configuration of the fluorimeter incorporates these inexpensive filters and replaces the dichroic mirrors with $\varphi^{1 / 2}$ " beam splitters, reducing the overall cost of the system from $\$ 830$ to $\$ 450$ USD.

Representative images of the assembly are shown for the first configuration in Figure 1 and Figure 2, but analogous images for the second configuration can be found in Supplemental

File 6. To avoid the need for specialized optical alignment, the optical system has designated areas to place each optical component and can be made with a relatively lowend 3D printer, allowing for widespread use of the design. The only differences in construction and assembly for the two configurations are the files used for 3D printing and the optical components placed into the enclosure. The external dimensions of the 3D printed enclosure for both systems are the same. A cost comparison of the two systems is shown in Table 1.

As shown in Figure 1A, to maintain a small form factor, the fluorimeter consists of $\Phi \frac{1}{2} 2^{\prime \prime}(\sim 12.5 \mathrm{~mm})$ optics, coupled with compact illumination and detection that are placed to measure signal through the top of the PCR tube. The system in Figure 1 is designed to detect dyes with peak excitation and emission wavelengths near $490 \mathrm{~nm}$ and $525 \mathrm{~nm}$, respectively, including FITC and closely related dyes such as SYBR and SYTO-9, which are commonly used as reporters in realtime nucleic acid amplification reactions ${ }^{7,8}$. The excitation source, optical filters, and detector can readily be substituted for components compatible with different fluorescent dyes as desired. Nucleic acid amplification reactions are typically performed in PCR tubes, and the fluorimeter is designed to be placed atop any commercially available heat block that holds PCR tubes (Figure 1D) allowing for real time monitoring of isothermal reactions. Appropriate heat blocks are available in 
most biomedical laboratories and can be purchased for less than $\$ 500$ USD.

The use of single-board computers to provide a low-cost, point of care alternative for controlling imaging technologies has been previously demonstrated ${ }^{9}$. Building off that work, in this protocol a single-board computer-powered graphical user interface (Figure 1D) is used to facilitate real-time data logging and display of results at the point of care, eliminating the need for a laptop computer to process or visualize data. Fluorescence measurements were transferred through $\mathrm{I}^{2} \mathrm{C}$ protocol from the light sensors to a microcontroller, and then made available to the single-board computer through serial communication. Electrical connections for illumination and data transfer were provided through simplified wiring and soldering on miniaturized breadboards, negating the need for specialized printed circuit boards (PCBs). The software required to run the fluorimeter is available through opensource software frameworks and the code required to run the device is provided in the supplemental coding files. The complete fluorimeter can be assembled for between $\$ 450$ to $\$ 830$ USD, and results show that it provides accurate and reliable fluorescence measurements to monitor real-time isothermal amplification of nucleic acids.

\section{Protocol}

\section{Preparation steps: 3D printing and soldering}

NOTE: The optical system described in this protocol is designed for a standard dry block heater.

1. To create the first configuration, $3 D$ print the $C A D$ files provided as Supplemental Files 1, 2, and $\mathbf{3}$ respectively:
2. To create the second configuration, $3 D$ print the $C A D$ files provided as Supplemental Files 3, 4, and 5, respectively:

NOTE: These parts are designed to be printed with supports. In this guide, a black polycarbonate filament is used that can maintain its form after being subjected to temperatures up to $110{ }^{\circ} \mathrm{C}$. In general, any material that can be heated to the temperature of the desired isothermal reaction without significant deformation can be used. To minimize the effect of internal reflections and ambient light interference, a material that is black or another dark color is recommended.

3. Prepare the two light-to-digital sensor evaluation modules for parallel monitoring of two samples. On one of the sensor test boards, remove the R4 resistor, and solder a jumper wire from the right pad of the R4 area on the PCB to the top pad in the R1 area on the PCB. This will change the $\mathrm{I}^{2} \mathrm{C}$ address of the sensor, thus allowing simultaneous measurement of both sensors.

NOTE: The sensor used consists of two PCBs: a USB adapter board and a sensor test board containing the light sensor; only the sensor test board is needed for this device.

4. Solder wires to each of the two Light Emitting Diodes (LEDs). Connect a red wire (positive) to the pad labeled "1" on the LED and a black wire (negative) on the pad labeled "2" on the LED. Apply a thin layer of thermal adhesive on the back of the LED, place the LED on the top of an end cap, and wait until the thermal adhesive cures. On the other side of the end cap, add a heat sink. NOTE: When testing the LEDs before they are sealed in the enclosure, ensure to wear a proper blue light blocking eye protection. 
5. To create the second configuration, cut two 1/4-inch diameter circles from a blue excitation foil sheet and four 1/4-inch diameter circles from a yellow emission foil sheet with scissors or a razor blade.

6. Press an M2.5 hex shaped insert into each of the four holes on the slanted portion of the 'LCD_Screen_Holder.stl' part.

\section{Optical assembly}

1. Place a $3 / 16$-inch-long $4-40$ threaded insert into the hole on the top of the 'Optics_Enclosure_Bottom.stl' part. Place a 1/4-inch-long 4-40 threaded insert into all other holes of the 3D printed part as shown in Figure 2A.

2. Insert the sensor test board into the top cavity of the housing, with the five pins facing towards the top and closest to the center axis of the device. Secure with a 3/16-inch-long 4-40 screw through the hole on the sensor test board (Figure 2B).

3. Place one of the $20 \mathrm{~mm}$ focal length lenses in the section below the sensor test board with the convex side facing towards the bottom of the device and away from the test board (Figure 2C).

4. To create the first configuration, place the long pass filter into the next section below the $20 \mathrm{~mm}$ focal length lens (Figure 2D) placed in the previous step. To create the second configuration, place two yellow emission filter foils into the section below the lens.

5. To create the first configuration, place the dichroic mirror into the diagonal section near the center of the encasement while observing the filter orientation specified by the manufacturer (Figure 2E). To create the second configuration, place the beam splitter into the diagonal section. No specific orientation is needed for the beam splitter.

6. Place a second $20 \mathrm{~mm}$ focal length lens into the section below the dichroic mirror (or beam splitter, depending on configuration) with the convex side pointing towards the top of the device (Figure 2F).

7. To create the first configuration, place the excitation filter in the section to the right of the dichroic mirror, making sure the arrow points towards the dichroic mirror (Figure 2G). To create the second configuration, place one blue excitation filter foil into the section to the right of the beam splitter.

8. Place the $15 \mathrm{~mm}$ focal length lens to the right of the excitation filter with the convex side facing the dichroic mirror (Figure 2H).

9. Place an LED into the remaining section of the print, with the LED facing towards the dichroic mirror (or beam splitter, depending on configuration). Ensure the two wires leading from the LED are inserted into the recessed channels so that the print will close tightly.

10. Repeat steps 2.3-2.9 for the other side of the 3D printed part (Figure 2I).

11. Close the empty side of the print on top of the print with the optical components by placing the extruded portions of the top half of the encasement into the recessed grooves of the bottom half of the encasement. Secure the two printed parts together with 3/8-inch-long 4-40 screws (Figure 2J).

NOTE: If the two printed parts are not completely closed, stray excitation light can escape from the optical housing. Ensure proper blue light blocking eye protection is worn until a proper seal is achieved. Reseal the enclosure until no excess light escapes. 


\section{Electronics and touchscreen assembly}

1. Connect the two mini breadboards together, and then place the microcontroller onto one of the breadboards. Ensure that the microUSB port of the microcontroller faces outwards.

2. To connect LED modulation, connect the CTL pin of the LED (+) driver to a digital pin of the microcontroller and the LED (-) pin of the LED driver to a GND pin of the microcontroller.

3. Remove the plastic covers on the back of the breadboards. Press the adhesive backing of the breadboards to the $3 \mathrm{D}$ printed part to attach the combined breadboards to the inside of the back portion of the 'LCD_Screen_Holder.stl' printed part.

4. Secure the liquid crystal display (LCD) screen holder with the assembled breadboards inside to the optical enclosure assembled in section 2 with one-inch-long 4-40 screws.

5. To connect the LED power supply, connect the LED (+) pin of the LED driver to the positive wire of the first LED. Connect the negative wire of the first LED to the positive wire of the second LED on the breadboard. Connect the negative wire of the second LED to the LED (-) pin of the LED driver.

NOTE: The order of first or second LED is arbitrary.

6. To connect the LED driver power supply, connect the positive and negative wires of the $10 \mathrm{~V}$ power supply to the VIN+ and VIN- pins of the LED driver, respectively. (A barrel jack to two-pin adapter was used.)

7. Connect the sensor test board power supply and data transfer. Only four pins on the sensor test board are used: the SCK, SDA, VDUT, and GND. Take a 4-pin female to male jumper wire and connect those pins on the light-todigital sensor test boards to the mini breadboard through the gap in the top right of the LCD Holder print.

8. On the breadboard, ensure connections between the following are in place: the $3.3 \mathrm{~V}$ pin of the microcontroller and the VDUT pin of both test boards; the GND pin of the microcontroller and the GND pin of both test boards; the analog 4 (A4) pin of the microcontroller and the SDA pin of both test boards; and the analog 5 (A5) pin of the microcontroller and the SCK pin of both test boards.

NOTE: Because $\mathrm{I}^{2} \mathrm{C}$ communication is used for the light sensors, the SCK and SDA pins of both sensors can both be routed to the same pins of the microcontroller.

9. Secure the single-board computer onto the LCD Screen holder with four M2.5 screws. Ensure that the HDMI and power adapter ports of the single-board computer face upwards and the single-board computer is centered on the 3D printed part.

10. Connect the touchscreen display to the single-board computer as per the touchscreen instructions, and then connect the HDMI port of the single-board computer to the touchscreen's HDMI port.

\section{Software installation}

1. Install and use the web editor to upload the custom sketch "MiniFluorimeter_2Diode.ino" provided in Supplemental Coding File 1 onto the microcontroller. Ensure the "ClosedCube OPT3002" library is installed using the Library Manager.

2. Change the variable led_A_pin to the number of the digital pin used in step 3.3 (Electronics and touchscreen assembly section). 
3. Adjust the number of milliseconds the LED is turned on when acquiring fluorescence measurements by changing the value of the variable ExposureTime. Adjust the number of milliseconds between LED exposures by changing the value of the variable led_A_Interval.

4. Change the variable led_Power to a number between zero and one to adjust the brightness of the LEDs during exposures. Zero gives the maximum amount of brightness and one gives the lowest amount of brightness.

5. Turn on the ability to control the display via touchscreen by following the manufacturer's instructions provided with the 3.5-inch display.

NOTE: If desired, the 3.5-inch screen can be used as a monitor without touchscreen capabilities, and a keyboard and mouse can be attached to the USB ports of the single-board computer for control of the single-board computer.

6. Download the "MiniFluorimeter_2Diode_GUI.py" file from Supplemental Coding File 2 to a desired location on the single-board computer.

7. Ensure a working version of Python is installed on the single-board computer. Python 3.7 was used in the provided Python module, but any stable Python version could be used with appropriate changes to the provided script. Install the libraries needed for the Python program onto the single-board computer.

8. Change the variable measurement_time to the length of time desired to take measurements. The program ends acquisition and closes after the desired time is elapsed. The GUI also allows acquisition to be ended through a button on the user interface.
9. Change the variable serialPort to the serial address of the connected microcontroller.

\section{Recording real-time fluorescence data}

1. Turn on the commercial heat block and allow it to reach the desired temperature.

2. Power the single-board computer with a standard $5 \mathrm{~V}$ power supply provided with most single-board computer purchases. Connect the single-board computer to the microcontroller with a microUSB to USB cable.

3. Using the touchscreen, open the provided Python script. Change the measurement_time and serialPort variables to the desired values. Change the variable outputFilepath to the name of the data file the program generates. Ensure the filename ends in '.xlsx'.

4. Place two PCR tubes containing the reactions to be monitored into the heat block. Ensure that the placement of the PCR tubes aligns with the optical channels of the fluorimeter once it is placed onto the heat block.

5. Place the fluorimeter atop the heat block with the PCR tubes centered between the four pegs extruding from each optical channel of the fluorimeter. For optimal measurements, ensure that the $3 \mathrm{D}$ printed fluorimeter is attached securely into the wells of the heat block.

6. Securely attach the fluorimeter, plug in the power supply adapter for the LEDs.

7. Use the touchscreen to start the Python program. A graphical user interface (GUI) appears on the LCD screen and measures the real-time fluorescence.

8. Observe the real-time fluorescence measurements over time for both PCR tubes that are shown to the user on the GUI. 
9. After the experiment time determined by the user has elapsed, acquisition ceases. View the measurements in the output data file saved in the user-defined location. To end the measurements early, click the button labeled "Stop Acquisition" on the user interface.

\section{Representative Results}

Once assembled, fluorimeter performance can be validated by measuring fluorescence from a dilution series of FITC dye.

In Figure 3A, measurements of FITC dye at concentrations of $0,20,40,60$, and $80 \mathrm{pg} / \mu \mathrm{L}$ prepared in $1 \times$ PBS on both channels of the first configuration of the fluorimeter are shown. Each sample was measured three times with an LED exposure of $1.5 \mathrm{~s}$ at $20 \mathrm{~s}$ intervals. Both channels of the fluorimeter show a linear response across the desired range.

Clinical applicability of the fluorimeter was further demonstrated by using the system together with a commercially available dry heat block to perform amplification with two isothermal amplification technologies: RPA and RTLAMP.

Figure 3B demonstrates the baseline-subtracted time course of fluorescence measured during $39^{\circ} \mathrm{C}$ amplification of $50 \mu \mathrm{L}$ of real-time RPA positive and negative control reactions for kit positive control DNA provided in a standard commercial kit and prepared per manufacturer's instructions. RPA reactions, which produce a relatively low level of fluorescence, were measured using the first configuration of the fluorimeter that achieves better suppression of excitation light.
Figure $3 \mathrm{C}$ demonstrates the time course measurement of a custom RT-LAMP assay at $65^{\circ} \mathrm{C}$ utilizing the N2, E1, and As1e primer sets described by Zhang et al ${ }^{10}$, and Rabe and Cepko ${ }^{11}$. RT-LAMP reactions produce a greater amount of fluorescence and were measured using the second, lower-cost fluorimeter configuration. Oligonucleotides were purchased and resuspended in $2 x$ TE buffer at a $1 \mathrm{mM}$ concentration. Forward inner primer (FIP) and backward inner primer (BIP) oligos were ordered with high performance liquid chromatography purification. Each primer set (N2, E1, and As1e) was combined to make $1000 \mu \mathrm{L}$ of a $25 \mathrm{x}$ mix as follows: $40 \mu \mathrm{L}$ of FIP, $40 \mu \mathrm{L}$ of BIP, $5 \mu \mathrm{L}$ of $\mathrm{F} 3,5 \mu \mathrm{L}$ of B3, $10 \mu \mathrm{L}$ of $\mathrm{LF}$, $10 \mu \mathrm{L}$ of $\mathrm{LB}$, and $890 \mu \mathrm{L}$ of $1 \mathrm{x}$ TE buffer. To assemble each RT-LAMP reaction, $1 \mu \mathrm{L}$ of each primer set was added to 0.5 $\mu \mathrm{L}$ of $50 \mathrm{x}$ fluorescent dye and $12.5 \mu \mathrm{L}$ of $2 \mathrm{x}$ master mix and the reaction volume was brought up to $20 \mu \mathrm{L}$ with nucleasefree water per manufacturer's instructions. The SARS-CoV-2 RNA Control was serially diluted in nuclease-free water to concentrations of 10,100 , or 1,000 copies per $\mu \mathrm{L}$, and 5 $\mu \mathrm{L}$ were added for a total reaction volume of $25 \mu \mathrm{L}$. The no target control (NTC) used in all experiments was nucleasefree water. RT-LAMP reactions were overlayed with $25 \mu \mathrm{L}$ of molecular biology-grade mineral oil.

RPA and RT-LAMP reactions were assembled in two wells of a $0.2 \mathrm{~mL}$ low-profile 8-tube PCR strip and capped with ultraclear flat caps. Each RPA and RT-LAMP reaction was run in triplicate. In all tests, the mini-fluorimeter successfully quantified the temporal increase of fluorescence levels associated with DNA amplification. 

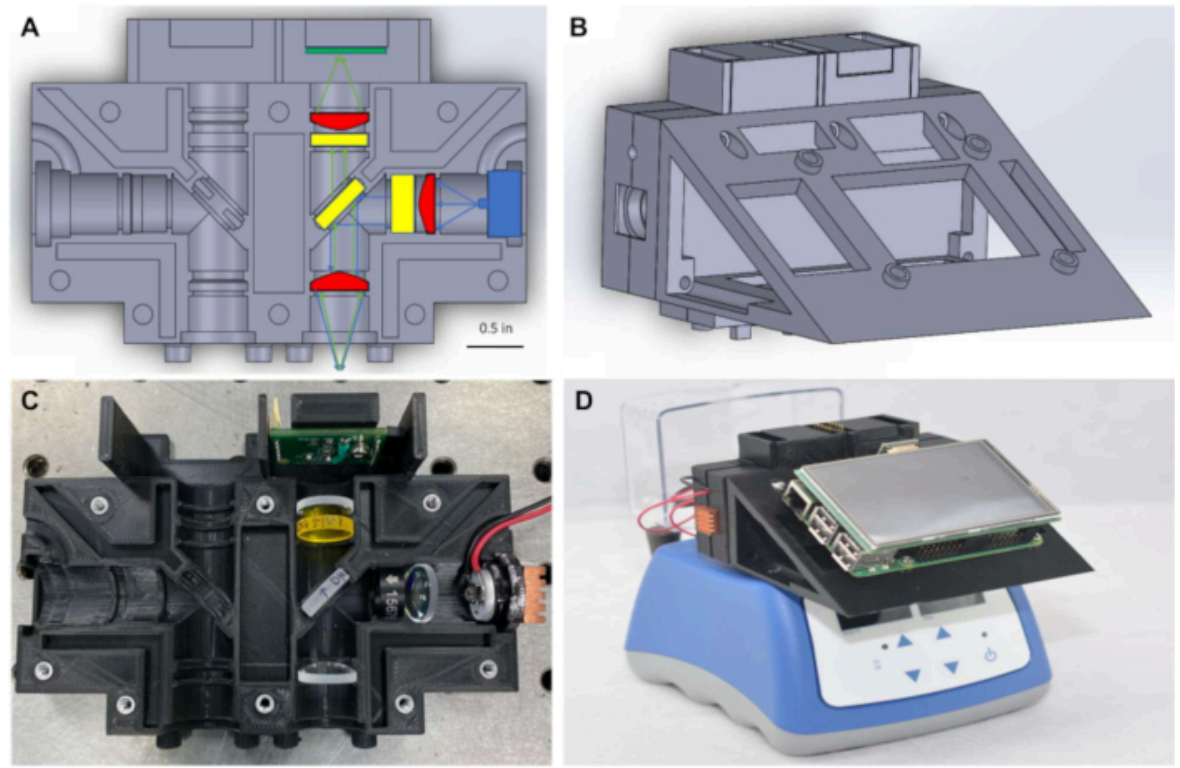

Figure 1: Optical housing and assembled miniature fluorimeter atop heat block. (A) Diagram of optical housing showing optical components placed in one detection channel. (B) Diagram of the first configuration of the miniature fluorimeter following assembly. (C) Photograph of the optical housing with optical components placed in one detection channel. (D) Photograph of assembled miniature fluorimeter placed on top of a commercially available heat block. Please click here to view a larger version of this figure. 

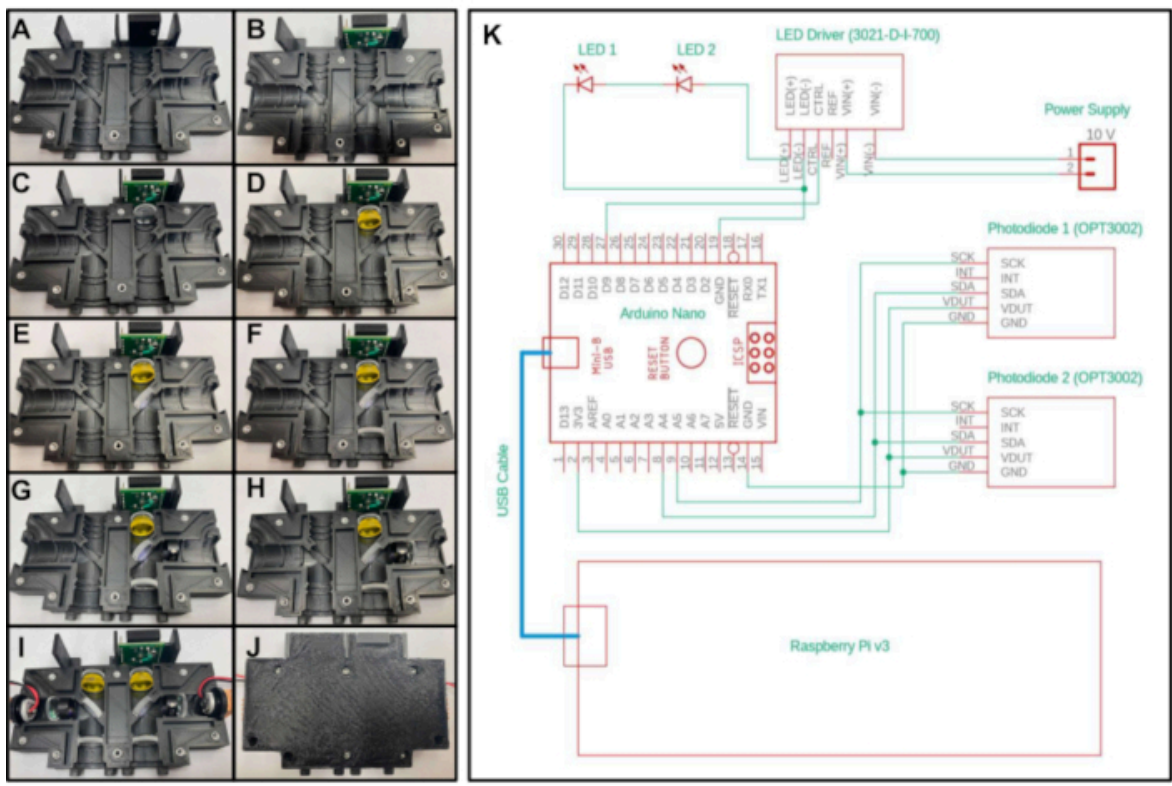

Figure 2: Assembly and electrical control diagram of the miniature fluorimeter. A-J) Step-by-step placement of the optical components in the 3D printed optical housing for the first system configuration. (K) Electrical diagram of the miniature fluorimeter for both configurations. Please click here to view a larger version of this figure. 
A

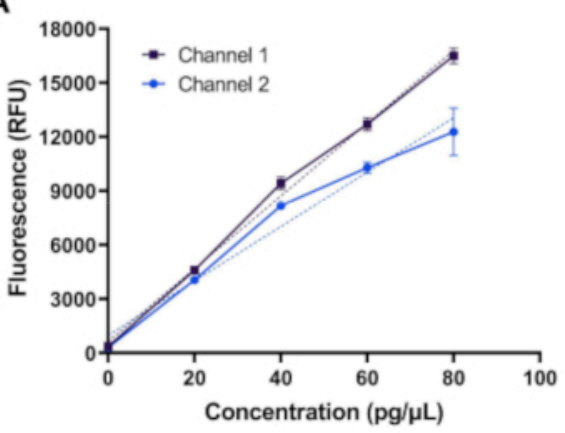

B

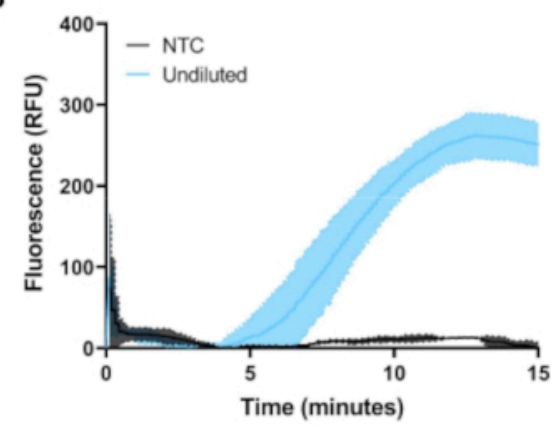

C

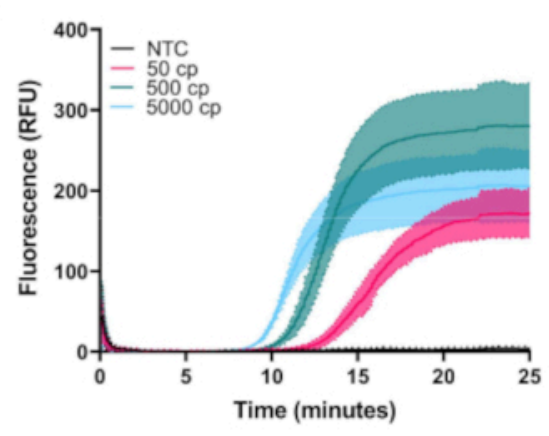

Figure 3: Representative measurements obtained with the miniature fluorimeter. (A) Measured fluorescence vs. FITC dye concentration in both channels shows linear response across the desired dynamic range. (B) Real-time fluorescence vs time for isothermal amplification of positive and negative controls of a commercially available kit. Amplification occurs as expected for the positive control. (C) Real-time fluorescence vs time for isothermal amplification of 50, 500, and 5000 copies of SARS-CoV-2 RNA and an NTC sample from a custom RT-LAMP assay. Amplification occurs as expected near the limit of detection of the assay. Please click here to view a larger version of this figure. 


\begin{tabular}{|c|c|c|c|c|}
\hline & \multicolumn{2}{|c|}{ System 1} & \multicolumn{2}{|c|}{ System 2} \\
\hline Item & Quantity & Total Price (USD) & Quantity & Total Price (USD) \\
\hline \multicolumn{5}{|c|}{ Optical Components } \\
\hline Lenses & 6 & 158.14 & 6 & 158.14 \\
\hline Mirrors & 2 & 244.56 & 2 & 60 \\
\hline \multirow[t]{2}{*}{ Optical Filters } & 4 & 200 & 6 & 5 \\
\hline & Subtotal & 602.7 & Subtotal & 223.14 \\
\hline \multicolumn{5}{|c|}{ Illumination and Detection } \\
\hline LEDs & 2 & 72.62 & 2 & 72.62 \\
\hline LED Driver & 1 & 11.49 & 1 & 11.49 \\
\hline \multirow[t]{2}{*}{ Photodiode } & 2 & 50 & 2 & 50 \\
\hline & Subtotal & 134.11 & Subtotal & 134.11 \\
\hline \multicolumn{5}{|c|}{ Electronics and Display } \\
\hline Arduino Nano & 1 & 20.7 & 1 & 20.7 \\
\hline Raspberry Pi & 1 & 35 & 1 & 35 \\
\hline LCD Screen & 1 & 25 & 1 & 25 \\
\hline Mini Breadboard & 1 & 4 & 1 & 4 \\
\hline \multirow[t]{2}{*}{ 10V Power Supply } & 1 & 8.6 & 1 & 8.6 \\
\hline & Subtotal & 93.3 & Subtotal & 93.3 \\
\hline \multicolumn{2}{|c|}{ Total Cost (USD) } & 830.11 & & 450.55 \\
\hline
\end{tabular}

Table 1: Cost comparison of the two configurations of the miniature fluorimeter.

Supplemental File 1: System1_Optics_Enclosure_Top.stl Please click here to download this file.
Supplemental File 3: LCD_Screen_Holder.stl Please click here to download this file.
Supplemental

File

System1_Optics_Enclosure_Bottom.stl, and Please click
2: Supplemental File 4: System2_Optics_Enclosure_Top.stl Please click here to download this file.

here to download this file. 


\section{Supplemental}

File

System2_Optics_Enclosure_Bottom.stl, and Please click here to download this file.

\section{Supplemental File 6: System2_BuildInstructions.pdf} Please click here to download this file.

\section{Supplemental Coding File 1: MiniFluorimeter_2Diode.ino Please click here to download this Coding File.}

\section{Supplemental Coding $\quad$ File MiniFluorimeter_2Diode_GUI.py Please click here to download this Coding File.}

\section{Discussion}

Described here is an open-source, low-cost, modular, portable fluorimeter for quantitative fluorescence detection of isothermal amplification reactions. Open-source projects facilitate rapid and inexpensive maintenance with readily available replacement parts and allow users the flexibility to adapt the system to their needs based on modular design. This protocol describes the process of assembling mechanical, optical and electrical components, and validating optical performance. Furthermore, the flexibility of the fluorimeter to monitor two different types of isothermal amplification assays with significantly different temperature, volume, and fluorescence requirements, RPA exo and RTLAMP, was demonstrated. RPA is performed at $39{ }^{\circ} \mathrm{C}$ in $50 \mu \mathrm{L}$ reactions that utilize a sequence-specific FAMtagged probe for fluorescence generation, while RT-LAMP is performed at $65{ }^{\circ} \mathrm{C}$ in a $25 \mu \mathrm{L}$ reaction volume and uses an intercalating dye to report the presence of the amplified DNA. Because fluorescence measurements are made through the top of PCR tubes with flat caps, the fluorimeter is able
: to detect fluorescence from both assay volumes, and heat requirements are only limited by the commercial heat block chosen. Furthermore, the fluorescence intensity produced in RT-LAMP is almost in the order of magnitude greater than that produced in RPA, due to the dye- versus probe-based methods of fluorescence signal generation. However, the dynamic range of the optical sensor chosen can detect and quantify both the signals, and baseline subtraction algorithms account for these differences to produce reliable fluorescence readings.

To facilitate technology dissemination and minimize potential maintenance cost, a modular design that is compatible with heaters that are widely available in different settings was employed. In the current protocol, a common dry block heater was used; the same optical and electrical design can be readily adapted for other commercially available heaters. If another dry block heater is to be used, minimal changes in the 3D housing design will be required. Specifically, the bottom pegs of the optical enclosure STL files must be modified to ensure proper alignment with the wells of other commercial heat blocks. While the enclosures shown in the examples were printed on a relatively low-end 3D printer (see Table of Materials), care should be taken to ensure the printer resolution and/or print tolerances are adequate to accommodate the optical components and threaded inserts. In the STL files provided, a tolerance of 0.01-0.02 inches was added on either side of the optical components in the radial and axial directions based on the dimensions specified by the manufacturer. This ensures all optical components fit securely within the print and that the enclosure completely blocks excess light from entering or escaping. To ensure a proper press fit for the threaded inserts, a similar tolerance 
of 0.01-0.02 inches was subtracted from the manufacturerprovided diameter in the CAD file.

RPA reactions were successfully monitored using the first fluorimeter configuration, while RT-LAMP reactions could be monitored using either configuration. The improved stray light rejection of the first configuration was necessary to monitor the low levels of fluorescence produced by the fluorogenic probe in RPA reactions. In contrast, RT-LAMP utilizes an intercalating dye for signal generation, resulting in a higher fluorescence intensity that is compatible with the lower dynamic range of the second configuration using photographic filter foils. Users should select the fluorimeter configuration that matches the fluorescence signal generating element-intercalating dye or fluorogenic probe-of their assay.

One limitation of this system is that heating is provided by a commercially available heat block powered through a standard wall outlet. This system could be further developed for use in areas lacking reliable access to electricity by incorporating portable and rechargeable battery packs as shown by other groups ${ }^{12}$. Another limitation is the relatively low throughput of the system, which allows for simultaneous fluorescence measurement of only two samples at a time. Multiple prints of the enclosure can be placed atop the same heat block to increase throughput; however, the light sensor used only has four unique $\mathrm{I}^{2} \mathrm{C}$ addresses. This restricts the maximum number of samples that can be simultaneously measured to four. A different light sensor with a larger number of unique $\mathrm{I}^{2} \mathrm{C}$ addresses is needed to further increase throughput.

\section{Disclosures}

The authors declare no conflicts of interest.

\section{Acknowledgments}

Special thanks to Chelsey Smith, Megan Chang, Emilie Newsham, Sai Paul, and Christopher Goh for their assistance with sample preparation. The authors thank Caroline Noxon for manuscript revision. Funding for this work was provided from the American people by USAID through an IAVI research grant CCID 9204 under award AID-OAA-A16-00032 between IAVI and USAID.

\section{References}

1. Daher, R.K., Stewart, G., Boissinot, M., Bergeron, M.G. Recombinase polymerase amplification for diagnostic applications. Clinical Chemistry. 62 (7), 947-958 (2016).

2. Yan, L. et al. Isothermal Amplified Detection of DNA and RNA. Molecular BioSystems. 10 (5), 970-1003 (2014).

3. Giuffrida, M.C., Spoto, G. Integration of Isothermal Amplification Methods in Microfluidic Devices: Recent Advances. Biosensors and Bioelectronics. 90, 174-186 (2017).

4. Gill, P., Ghaemi, A. Nucleic Acid Isothermal Amplification Technologies-A Review. Nucleosides, Nucleotides and Nucleic Acids. 27 (3), 224-243 (2008).

5. Richards-Kortum, R., Oden, M. Devices for LowResource Health Care. Science. 342 (6162), 1055-1057 (2013).

6. Katzmeier, F. et al. A Low-Cost Fluorescence Reader for in vitro Transcription and Nucleic Acid Detection with Cas13a. PLOS One. 14 (12): e0220091(2019).

7. Safavieh, M. et al. Emerging Loop-Mediated Isothermal Amplification-Based Microchip and Microdevice Technologies for Nucleic Acid Detection. ACS 
Biomaterials Science and Engineering. 2 (3), 278-294 (2016).

8. Monis, P.T., Giglio, S., Saint, C.P. Comparison of SYTO9 and SYBR Green I for Real-Time Polymerase Chain Reaction and Investigation of the Effect of Dye concentration on Amplifcation and DNA Melting Curve Analysis. Analytical Biochemistry. 340 (1), 24-34 (2005).

9. Parra, S. et al. Development of Low-Cost Point-of-Care Technologies for Cervical Cancer Prevention Based on a Single-Board Computer. IEEE Journal of Translational Engineering in Health and Medicine. 8 (4300210) (2020).

10. Zhang, Y. et al. Enhancing Colorimetric Loop-Mediated Isothermal Amplification Speed and Sensitivity with Guanidine Chloride. Biotechniques. 69 (3), 178-185 (2020).

11. Rabe, B.A., Cepko, C. SARS-CoV-2 Detection Using an Isothermal Amplification Reaction and a Rapid, Inexpensive Protocol for Sample Inactivation and Purification. Proceedings of the National Academy of Sciences of the United States of America. 117 (39), 24450-24458 (2020).

12. Snodgrass, R. et al. A Portable Device for Nucleic Acid Quantification Powered by Sunlight, a Flame or Electricity. Nature Biomedical Engineering. 2 (9), 657-665 (2018). 